\title{
Inversion Algorithms for GPS Downward Looking Occultation Data: Simulation Analysis
}

\author{
Ashraf MOUSA ${ }^{1}$ and T. TSUDA \\ Radio Science center for Space and Atmosphere, Kyoto University, Kyoto, Japan
}

(Manuscript received 14 March 2003, in revised form 10 November 2003)

\begin{abstract}
Downward looking (DL) GPS receivers from platforms within Earth's atmosphere (e.g. from a mountain top) can observe GPS satellites and produce an estimate of atmospheric refractivity profiles through the radio occultation technique. The main observations are the radio wave bending angles as a function of the impact parameter. The DL technique provides bending angles at both negative and positive elevation angles. Either a least-squares ray tracing method or the Abel inversion technique can be used for the retrieval of the refractivity from the bending angles. A least-squares method (LS) can be applied directly to the set of positive and negative elevation angles. It allows the retrieval of the refractivity to about $2 \mathrm{~km}$ above the receiver height. The Abel inversion operates on a profile of partial bending angles found by subtracting the positive elevation measurement from the negative ones with the same impact parameter.

This paper summarizes the promises and limitations of the two inversion techniques using simulated data. The analysis uses both dry and wet atmospheres from a climatological model and real radiosonde data. The results show that both LS and Abel techniques are capable of retrieving the refractivity. The paper also highlights the differences between the two algorithms and the limitations.
\end{abstract}

\section{Introduction}

Space-based radio occultation (RO) measurements using GPS receivers on low Earth orbiting (LEO) satellites provide accurate atmospheric refractivity profiles (Kursinski et al. 1997; Rocken et al. 1997; Wickert et al. 2001). The RO technique has been widely used in the study of planetary atmospheres (e.g., Fjeldbo et al. 1971). The basic idea behind the radio occultation is to measure how radio waves are bent by the refractive index gradients in an atmosphere. Assuming spherical symmetry of the atmosphere, this bending information can be inverted with an Abel inversion to produce a vertical profile of refractive index.

Corresponding author: Ashraf Mousa, Radio Science Center for Space and Atmosphere, Kyoto University, Gokasho, Uji, Kyoto 611-0011, Japan.

1 Present affiliation: National Research Institute of Astronomy and Geophysics, Helwan, Cairo, Egypt. E-mail: ashrafkm@yahoo.com

(C) 2004, Meteorological Society of Japan
RO measurements with a receiver inside the atmosphere, placed either on an airplane or a mountain top, for example, has been proposed by Zuffada et al. (1999). While GPS-LEO occultation data have the advantage of being global (one receiver in LEO can provide about 500 globally distributed occultation events per day), the sampling in any region is relatively sparse without a large number of orbiting receivers (e.g., Kursiniski et al. 1997). In contrast, a receiver located inside Earth's atmosphere can be used to provide data over specific areas of interest for the purpose of regional weather and climate studies.

A mountain-based or airborne receiver can track any GPS satellite as it sets or rises behind Earth's limb. Therefore, data can be collected at both negative and positive elevation angles relative to the receiver local tangent (local horizon). Every occultation will produce a profile of refractivity below the height of the receiver, with a diffraction-limited, vertical res- 
olution of $150-250 \mathrm{~m}$. An occultation with the receiver inside the atmosphere is known as a downward looking (DL) occultation.

Although fundamentally DL measurements are similar to the LEO measurements, it was originally thought that the limits of integration used in the Abel transform prevented its implementation when the receiver is inside the atmosphere (Zuffada et al. 1999). In fact, it is possible to use an Abel inversion for the DL case. The measurement geometry is similar to the one considered by Bruton and Kattawar (1997) when inverting solar occultation data.

This paper compares a least-squares raytracing technique and the Abel inversion method for DL retrievals. Section two introduces the GPS occultation theoretical considerations and highlights the basics of the inversion techniques. Section three describes the results of the inversions using simulated data. Basic conclusions and discussion are given in Section four.

\section{Downward looking occultations- theoretical considerations}

For each GPS occultation event, the data analysis chain from the measured phase delay to the derivation of the refractivity can be divided into two main steps:

1. Calculation of the atmospheric bending angle profile from the observed L1/L2 excess phase path time series (L1 and L2 are the phases of the two GPS carrier frequencies).

2. Retrieval of the refractivity profile from the atmospheric bending angles.

\subsection{Bending angle calculation}

In the geometric optics approximation, a ray passing through the atmosphere behaves according to Fermat's principle of least time. The ray travels along a curve defined by

$$
n r \sin (\varphi)=\text { constant } \equiv a,
$$

where $r$ is the distance from the origin of symmetry to a point on the ray path, $\varphi$ is the angle between the direction of $r$ and the tangent to the ray path, and $n$ is the refractive index at $r$ (Fig. 1). Equation (1) corresponds to Snell's law in polar coordinates for a spherically symmetric medium, and is known as Bouguer's formula. On this basis, a signal traveling in a spherically

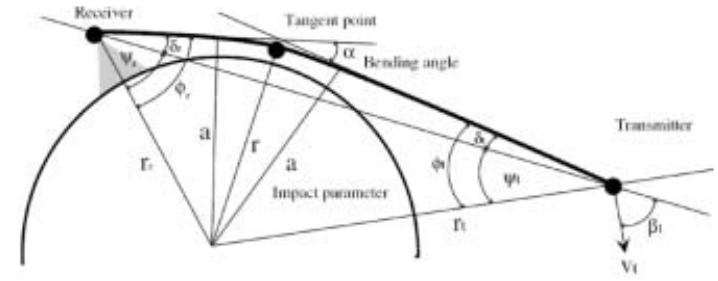

Fig. 1. A schematic diagram defining the geometrical variables for a GPS transmitter/receiver link. Note that the angle $\delta_{t}$ is the angle between the line connecting the transmitter and receiver and the ray tangent at the transmitter, $\delta_{r}$ is the angle between the line connecting the transmitter and receiver and the ray tangent at the receiver, $\psi_{t}$ is the angle between the line connecting the transmitter and receiver and the radius direction at the transmitter, $\psi_{r}$ is the angle between the line connecting the transmitter and receiver and the radius direction at the receiver, $\beta_{t}$ is the angle between the line connecting the transmitter and the receiver and the transmitter velocity vector $V_{t}$, and the angles $\varphi_{t}$ and $\varphi_{r}$ are the angles given before in equation 1 with the subscript $t$ and $r$ for transmitter and receiver respectively.

symmetric medium will bend by an angle $\alpha$ (Born and Wolf 1980)

$$
\alpha=-2 a \int_{a}^{\infty} \frac{1}{n \sqrt{\left(n^{2} r^{2}-a^{2}\right)}} \frac{d n}{d r} d r,
$$

where $a$ is the impact parameter of the ray.

The basic GPS data from which the bending angles $\alpha$ are derived are the L1 and L2 phase delays. From knowledge of the position of the transmitter $\left(r_{t}\right)$ and the receiver $\left(r_{r}\right)$ and their clocks (Fig. 1), the excess Doppler shift due to the atmosphere (compared to the Doppler shift in a vacuum) can be calculated. Then the bending angle is derived as a function of the impact parameter $(a)$ from the Doppler shift (for more details see, e.g., Hajj et al. 1996). For this step, the geometry of figure 1 is very important.

\subsection{Inversion scheme}

The profile of bending angles as a function of impact parameter is the input data for the inversion. The Abel inversion for DL uses the difference between the bending angle observed 
at negative and positive elevation angles, while the least squares method uses the observed bending angle directly. However, least squares method requires an a priori model above the receiver to constrain the solution. Here the basics of both the least squares and the Abel inversion methods are described.

\section{a. Abel inversion}

The Abel inversion has been used extensively in seismic and astronomical inversions, as well as planetary and Earth occultation data (e.g., Fjeldbo et al. 1971; Kursiniski et al. 1997). Starting with the bending angle determined from the GPS Doppler shift, equation (2) is inverted with Abel inversion to give the refractive index (e.g., Tricomi 1977)

$$
n(x)=\exp \left(\frac{1}{\pi} \int_{a}^{\infty} \frac{\alpha(a)}{\sqrt{\left(a^{2}-x^{2}\right)}} d a\right),
$$

where $x=n r$ is the refractional radius.

Unlike the LEO case, in DL occultations there may be significant ray bending along sections of the path above the receiver position, so the bending will not be equal on both sides of the tangent point. However, Bruton and Kattawar (1997) noted that when the receiver is within the atmosphere, it is possible to observe rays at both positive and negative elevations. These refer to rays that intersect the receiver from above and below the local tangent. They also pointed out that, assuming spherical symmetry, for every negative elevation ray with bending angle $\alpha_{n e g}$, there is a corresponding positive elevation value $\alpha_{P}$ with the same impact parameter value. Subtracting $\alpha_{P}$ from $\alpha_{\text {neg }}$, gives the partial bending angle $\alpha^{\prime}(a)$

$$
\alpha^{\prime}(a)=\alpha_{n e g}(a)-\alpha_{P}(a)
$$

where $\alpha^{\prime}(a)$ is the bending that occurs along the section of path below the receiver. By definition, as the tangent point approaches the receiver distance, $r_{r}$, the partial bending approaches zero.

The partial bending angle $\alpha^{\prime}(a)$ can be written as:

$$
\alpha^{\prime}(a)=-2 a \int_{a}^{n\left(r_{r}\right)^{*} r_{r}} \frac{d \ln (n) / d x}{\sqrt{\left(x^{2}-a^{2}\right)^{2}}} d x
$$

where $x=n r, n\left(r_{r}\right)$ is the refractive index at the receiver position and $r_{r}$ is the receiver position.
Equation (5) can be inverted with,

$$
n(x)=n\left(r_{r}\right) \exp \left(\frac{1}{\pi} \int_{x}^{x\left(r_{r}\right)} \frac{\alpha^{\prime}(a)}{\sqrt{\left(a^{2}-x^{2}\right)}} d a\right),
$$

where $x\left(r_{r}\right)$ is the refractional radius at the receiver position.

\section{b. Ray tracing inversion technique}

The observation vector is composed of a set of $m_{p}$ positive and $m_{n}$ negative elevation bending angle data. Since each bending measurement at negative elevation is heavily weighted by the atmospheric structure at the layer where the tangent point resides, the atmospheric structure below the height of the receiver is expected to be strongly constrained by the bending measurements (Mousa and Tsuda 2001). However, as the elevation angle increases, the data become strongly correlated, and we must rely on other a priori information to obtain a unique solution for the atmosphere at high altitudes. For this a priori information we introduce $m_{c}$ refractivities taken from a climatological model above the receiver. These independent data are added as constraints and the problem becomes a constrained least-squares calculation.

The atmosphere is thus modeled as a set of concentric layers of specified thickness, with refractivity varying exponentially as a function of radius with a fixed scale height for each layer. This exponential model consists of $m$ layers, with $m=m_{p}+m_{n}+m_{c}$. The model is constrained such that the refractivity (but not its derivative) is continuous across the boundaries of different layers. Thus, refractivity can be formulated as a function of the radius $r$ as:

$$
\begin{aligned}
N(r)= & N_{\text {norm }} \exp \left(-\frac{r-R_{j}}{H_{j}}\right) \prod_{i=j}^{J_{\text {norm }}-1} \exp \left(+\frac{\Delta_{i}}{H_{i}}\right) \\
& j<j_{\text {norm }} \\
N(r)= & N_{\text {norm }} \exp \left(-\frac{r-R_{j}}{H_{j}}\right) \prod_{i=j_{\text {norm }}}^{J-1} \exp \left(-\frac{\Delta_{i}}{H_{i}}\right) \\
& j>j_{\text {norm }}, \\
N(r)= & N_{\text {norm }} \exp \left(-\frac{r-R_{j}}{H_{j}}\right) \quad J=j_{\text {norm }}, \quad(7)
\end{aligned}
$$

where $R_{j}$ is the lower boundary of the $j^{\text {th }}$ layer, $\Delta$ and $H_{i}$ are the $i^{\text {th }}$ layer thickness and scale 
height, respectively. $N_{\text {norm }}$ is a normalized refractivity; taken as the refractivity value at the lower boundary of an arbitrary layer, referred to as the normalized layer. Initial values of the $H_{i}$ are obtained from the a priori model. Note that the domain of the radius $r$ for each part of equation (7) is given by

$$
R_{j} \leq r \leq R_{J+1}
$$

The inversion consists of finding the optimal set of scale heights and an overall $N_{\text {norm }}$ that best fit the measured bending angles and the a priori constraints, weighted by their respective measurement uncertainty. For the simulations, bending angles are calculated using numerical integration of equation (2) (Zuffada et al. 1999). The error in the refractivity, used as constraints above the receiver position, is assumed to have a Gaussian distribution with standard deviation equal $0.05 \mathrm{~N}$. The errors in bending angles are also assumed to be random with a standard deviation given by $0.01 \alpha+10^{-5}$ radians. Because the problem is highly nonlinear, a few iterations are required before a solution is reached.

\section{Simulation results}

In order to compare the two algorithms' ability to retrieve the refractivity when a receiver is inside the atmosphere, we constructed three sets of simulated measurements; a dry and a wet case from the MSIS-90 model atmosphere (Mass-Spectrometer-Incoherent-Scatter model, which describes the temperature and densities in Earth's neutral atmosphere from ground to thermospheric heights; Heydin 1991) and a wet case with some vertical variability given by a radiosonde profile. The receiver is assumed to be stationary at $3.8 \mathrm{~km}$ altitude and tracking GPS satellite signals at both positive and negative elevations.

In all cases, starting with a model refractivity to be the truth, a set of rays linking the transmitter to the receiver were constructed with specified tangent heights, ranging from the Earth's surface to the receiver's height. In a similar fashion, a set of rays linking the GPS satellite to the receiver was constructed to represent positive elevation angles above the receiver horizon. Like the negative measurements, the positive measurements have impact parameter given by equation (1), where $r$ is the
Percentage of retrieval Error (Dry case)

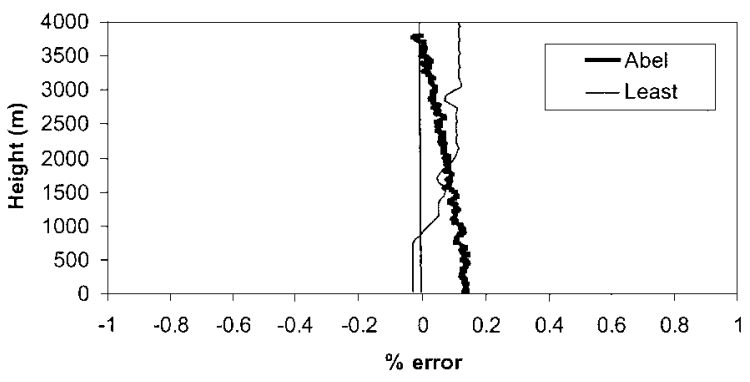

Fig. 2. Refractivity retrieval error (\%) for the dry model case. The receiver is at $3.8 \mathrm{~km}$ height.

\section{Percentage of Retrieval Error (Wet Case)}

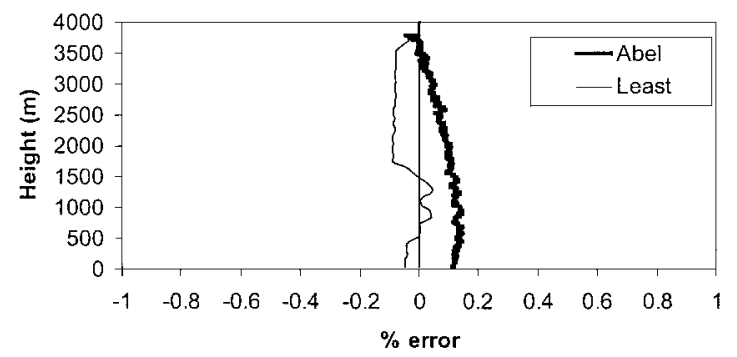

Fig. 3. Refractivity retrieval error (\%) for the wet model case.

radius of the receiver but $\varphi$ is between $90^{\circ}\left(0^{\circ}\right.$ elevation) and $180^{\circ}$ (zenith).

We start the simulation by calculating the bending angle as a function of impact parameter using the refractivity profile. Then random errors of zero mean are added to the calculated bending angles (standard deviation, $\mathrm{SD}=$ $0.01 \alpha+10^{-5}$ radians). This random error is intended to include the error due to the spherical symmetry approximation and to receiver thermal noise. Next we used this profile of bending angle as input to the two inversion methods. The errors of the inversion, height range of the results as well as the computer CPU time are considered in the comparison. Results are summarized in figures 2 through 5 in terms of the inversion errors for both cases.

The results show that the accuracies of both inversion methods are similar. The relative errors are always better than $0.2 \%$ (as is clear from figures two through four). The leastsquares method requires much more CPU time 
Percentage of retrieval Error. (radioSonde)

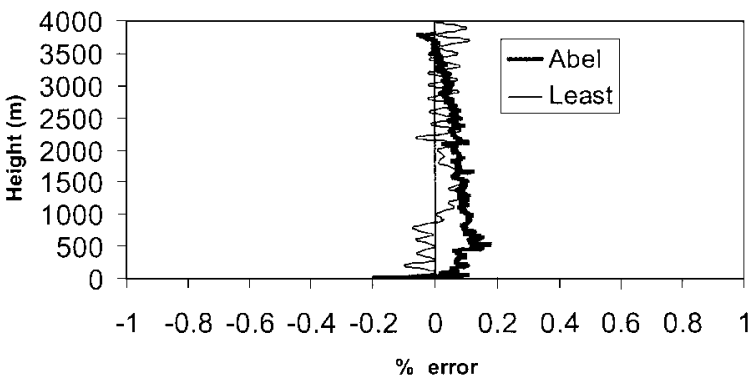

Fig. 4. Refractivity retrieval error (\%) for the radiosonde case.

Percentage of retrieval error (Biases)

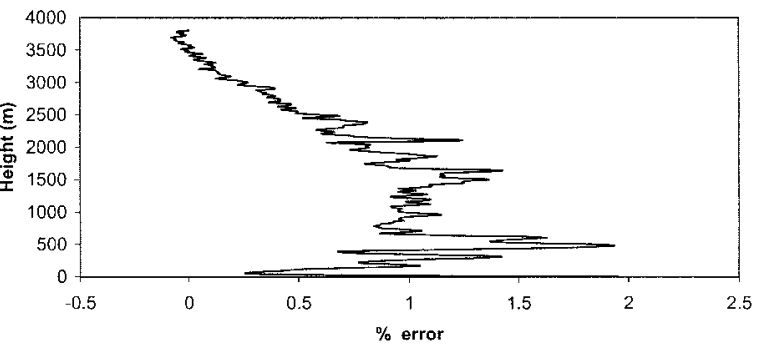

Fig. 5. Refractivity retrieval error (\%) for the radiosonde case for the Abel inversion. Elevation-dependant bias errors are added to the bending angles (about $10 \%)$.

than the Abel inversion (the ratio is about 60 to one on a P4 PC running Windows XP). In addition, the least-squares inversion requires an a priori model of refractivity, which is not required by the Abel inversion. However, the least-squares method can estimate the refractivity to about $2 \mathrm{~km}$ above the receiver positions in addition to elevations below the receiver, while the Abel method can only estimate the refractivity below the receiver.

We also compared the effect of biases in the bending angles on the two methods. A constant bias makes no difference at all for the Abel inversion. However, a constant bias greater than $1.454 * 10^{-4}$ radian $(30 \mathrm{sec})$ in the bending angle prevents the convergence in the least squares method. An elevation-dependent bias (the value of the bias is taken as zero at the zero degree elevation angle and increases as a linear function of the elevation angle until it
Table 1. Overall comparison of both Least squares and Abel inversions.

\begin{tabular}{|l|l|l|}
\hline Items & \multicolumn{1}{|c|}{ Least Squares } & \multicolumn{1}{|c|}{ Abel } \\
\hline $\begin{array}{l}\text { Refractivity } \\
\text { Model }\end{array}$ & needed & Not needed \\
\hline Accuracy & $\begin{array}{l}\text { Better than } \\
0.25 \%\end{array}$ & $\begin{array}{l}\text { Better than } \\
0.2 \%\end{array}$ \\
\hline $\begin{array}{l}\text { Constant } \\
\text { bias }\end{array}$ & $\begin{array}{l}\text { No convergence } \\
\text { if }>30 \text { arc second } \\
\left(1.454 * 10^{-4}\right. \\
\text { radians })\end{array}$ & $\begin{array}{l}\text { No problem } \\
\text { at all }\end{array}$ \\
\hline $\begin{array}{l}\text { Elev. Depen. } \\
\text { bias }\end{array}$ & $\begin{array}{l}\text { No convergence } \\
\text { CPU time }\end{array}$ & Few errors \\
\hline $\begin{array}{l}\text { Height } \\
\text { range }\end{array}$ & $\begin{array}{l}\sim 2 \mathrm{~km} \text { above } \\
\text { receiver }\end{array}$ & $\begin{array}{l}\text { Below } \\
\text { receiver }\end{array}$ \\
\hline
\end{tabular}

reaches $10 \%$ of the bending angle at the lowest elevation angle) prevents convergence for the least-squares method. Table 1 summarizes the limitations and promises of the two methods.

\section{Conclusions}

A least-squares and Abel inversion techniques are compared for retrieving DL GPS occultation data. Occultation data are simulated using profiles from a climatological model (MSIS-90) and radiosondes.

Both the least-squares and Abel inversions can retrieve the refractivity with a very good accuracy; the relative errors are better than $0.2 \%$ for the observational error model used in this study. The least-squares method can extend the height range to a few kilometers above the receiver but it requires an a priori refractivity model. However, the Abel inversion does not require an a priori model. The Abel inversion is also less sensitive to biases in the data.

\section{References}

Born, M. and E. Wolf, 1980: Principles of Optics. $6^{\text {th }}$ ed., Pergamon, 952pp.

Bruton, W.D. and G.W. Kattawar, 1997: Unique temperature profiles for the atmosphere below an observer from sunset images. Appl. Opt 36, $6957-6961$.

Fjeldbo, G., A.J. Kliore, and V. Eshleman, 1971: The neutral atmosphere of venus studied with 
the mariner $V$ radio occultation experiments. Astron. J., 76, 123-140.

Hajj, G.A., E.R. Kursiniski, W.I. Bertiger, S.S. Leroy, T. Meehan, L.J. Romans, and J.T. Schofield, 1996: Initial results of GPS-LEO occultation measurements of Earth's atmosphere obtained with GPS/MET experiment. Proceedings of Symp. on GPS Trends in Precise Terrestrial, Airborne, and Spaceborne Applications, Springer-verlag, 144-153.

Hedin, A.E., 1991: Extension of the MSIS Thermospheric Model into the Middle and Lower Atmosphere. J. Geophys. Res., 96, 1159-1991.

Kursinski, E.R., G.A. Hajj, K.R. Hardy, J.T. Schofield, and R. Linfield, 1997: Observing Earth's atmosphere with radio occultation measurement using the Global Positioning System. J. Geophys. Res., 102, 23429-23465.

Mousa, A.K. and T. Tsuda, 2001: Retrieval of key climate variables using occultation geometry of a mountain top GPS receiver. Proceedings of ION GPS 2001, 1117-1126.

Rocken, C., R. Anthes, M. Exner, D. Hunt, S. Sokolovskiy, R. Ware, M. Gorbunov, W. Schreiner, D. Feng, B. Herman, Y.-H. Kuo, and X. Zou, 1997: Analysis and validation of GPS/MET data in the neutral atmosphere. J. Geophys. Res, 102, 29849-29866.

Tricomi, F.G., 1977: Integral Equations. Dover, 238pp.

Wickert, J., C. Reigber, G. Beyerle, R. Konig, C. Marquardt, T. Schmidt, L. Grunwaldt, R. Galas, T.K. Meehan, W.G. Melbourne, and K. Hocke, 2001: Atmospheric sounding by GPS radio occultations: first results from CHAMP. Geophys. Res. L, 28, 3263-3266.

Zuffada, C., G.A. Hajj, and E.R. Kursiniski, 1999: A Novel approach to atmospheric profiling with a mountain-based or airborne GPS receiver. $J$. Geophys. Res., 104, 24435-24447. 